\title{
Immunophenotyping pattern characterization in canine blood: towards a clinical application
}

\author{
Leticia G. León ${ }^{1}$ Fatima Cruz Lopez ${ }^{2}$, M. Luisa Fermín ${ }^{3}$, Guillermo Mejías ${ }^{4}$, \\ Elisabeth $\mathrm{Kremmer}^{5}$, Concepción Tejero ${ }^{4}$
}

${ }^{1}$ Centre for Biomedical Research of the Canary Islands (CIBICAN) ULL, Institute of Biomedical Technologies, Tenerife, Spain

${ }^{2}$ Complutense University of Madrid, Veterinary Hospital Clinic, Laboratory of Clinical Pathology, ${ }^{3}$ Veterinary Faculty, Department of Animal Surgery and Medicine, ${ }^{4}$ Department of Biochemistry annd Molecular Biology, Madrid, Spain

${ }^{5}$ Institute for Molecular Immunology, Helmholtz Zentrum München, Munich, Germany

Received July 13, 2015

Accepted May 2, 2016

\begin{abstract}
Immunophenotyping is a widely used method for a precise diagnosis and classification of haematopoietic neoplasia in human beings and also in dogs. The gold standard for cell preparation is density gradient centrifugation of mononuclear cells. Alternatively, another way to separate human leukocytes is carrying out whole blood lysis. The aim of this study was to validate whole blood lysis as an alternative method in clinical veterinary procedures using an immunophenotype panel of leukocytes designed by our group. Flow cytometry study of adult canine leukocytes subset groups, using whole blood lysis or mononuclear cells tested against an array of canine leukocyte antibodies were done. Besides differential white blood cell counts were done. Also immunophenotyping studies in whole blood samples stored at $4{ }^{\circ} \mathrm{C}$ for $48 \mathrm{~h}$ were performed. The Coefficient Variation values were less than $20 \%$, for most of the comparison. Consistent results were observed in phenotyping canine peripheral blood leukocytes. Stability results indicated that whole blood samples might be stored for $48 \mathrm{~h}$ without a significant difference in the data compared to samples processed immediately after blood collection. This study shows that whole blood lysis represents an efficient and quick alternative for canine leukocyte preparation. In addition, samples can be analysed immediately or stored for $48 \mathrm{~h}$ without a significant difference between them. This is relevant for veterinary medicine considering the lack of facilities in many laboratories to process samples.
\end{abstract}

Flow cytometry, peripheral blood, leukocyte subsets, long-term stability

Immunophenotyping is a widely used method, complementing morphology, cytochemistry, clonal assays and karyotype study to make more precise diagnosis and classification of haematopoietic neoplasia in animals as well as humans. More precisely, dogs are relevant for immunological investigation since spontaneous immune-mediated and autoimmune diseases occur at high prevalence in canine population (Kolb et al. 1997).

Flow cytometry is a powerful tool used for diagnostic purposes. Evaluation of surface and intracellular antigens with a panel of antibodies allows identification of specific cell lineages as well as the maturation stage in malignant diseases (Weiss 2002). However, in contrast to humans, the small number of commercial mAbs available for dogs makes the standardization studies for specific diseases more difficult. Good Laboratory Practices recommend validation of sample preparation by flow cytometry (Zeigler et al. 2013). Peripheral blood, bone marrow, or tissue specimens should be processed to contain a cell suspension, eliminating erythrocytes, to give an optimal cell concentration for monoclonal staining (Owens et al. 2000).

The gold standard for mononuclear cell preparation is density gradient centrifugation (Van Dogen et al. 2003). Alternatively, another way to separate human leukocytes is 
carrying out whole blood lysis (Schwonzen et al. 2007). This method is more appealing because it is less time consuming, cheaper and requires less sample volume. The percentages of canine lymphocyte subsets obtained by these two methods did not show significant differences (Faldyna et al. 2001) and previous observation did not find significant differences between the two methods (De Paoli et al. 1984; Mansour et al. 1990).

Canine Leukocyte Antigen Workshop (CLAW), examined lymphocyte populations in healthy dogs. Many similarities between the dog and other animals have been demonstrated, establishing canine antibody equivalents of CD3, CD5, CD4, CD8 used to label lymphocyte T-cell subsets from peripheral blood $(\mathrm{McS} w e e n e y$ et al. 1998; Hardy et al. 2013). Furthermore, in healthy beagle dogs over 8 years old, where tumour incidence becomes higher, the immune status of CD3, CD4, CD8 and CD21 could predict future morbidity and mortality (Watabe et al. 2012). By turn, the discovery of CD34 as an immature haematopoietic cell surface antigen and the production of anti CD34-monoclonal antibodies provided an important tool to study haematologic malignancies (Cobbold et al. 1994; McSweeney et al. 1998; Ostronoff et al. 2008; Williams 2008). In addition, CD4, CD5, CD8, CD21 and CD34 have been utilized as markers to study canine lymphoproliferative prognosis (Krause et al. 1996).

The goal of this study was to establish an immunophenotype panel of leukocytes (CD3, CD4, CD8, Dog10(CD8), Dog17(Pan T), CD21, CD34) from canine mononuclear cells (MWBC) and total white blood cells (TWBC) of clinically healthy dogs aged between 6-8 years. The verification of both methods will be beneficial for the analysis in haematological diseases in animals. A secondary aim was to validate Dog 17 (panT) and Dog 10 (CD8) as canine antigens. Future studies will include these specific markers, which will ultimately be used for diagnosis and prognosis of canine leukaemia or malignant lymphoma.

\section{Materials and Methods}

Animals

10 healthy Beagle dogs ( 5 males and 5 females) within the age range of 6-8 years were selected for this study. All dogs were clinically healthy and presented no abnormalities in haemogram and serum chemistry. All serology were negative against Ehrlichia and Leishmania spp. This study was approved by the Institutional Animal Utilization Committee for Investigation of the Complutense University of Madrid, and was conducted in accordance with the Spanish legislation for the care of experimental animals (RD 53/2013). All animals were housed and cared for at the animal research facilities of the Veterinary Teaching Hospital of the Complutense University of Madrid.

\section{Collection and processing of blood specimens}

Blood samples were drawn in the morning from fasted animals by venipuncture of the jugular/cephalic vein and were collected in EDTA tubes for immediate immunophenotyping and flow cytometry analysis or stored for $48 \mathrm{~h}$ at $4{ }^{\circ} \mathrm{C}$ for the stability study.

\section{Cell preparation}

Blood samples were processed after surgery to obtain the mononuclear white blood cells (MWBCs) and the total white blood cells (TWBCs) free of erythrocytes. In both cases, only cellular suspensions with $>95 \%$ of viable cells were used.

MWBCs were obtained by blood centrifugation with Ficoll-Paque ${ }^{\mathrm{TM}}\left(\mathrm{d}=1.077 \mathrm{~g} / \mathrm{cm}^{3}\right)(\mathrm{GE}$ Healthcare, Uppsala, Sweden). Further, mononuclear cells were collected and washed with $10 \mathrm{ml}$ of MACS buffer (Miltenyi Biotech, Galdabach Germany). Finally, the cell pellet was suspended in $1 \mathrm{ml}$ of MACS buffer.

TWBCs were obtained by erythroid lysis. Well-mixed blood $(1 \mathrm{ml})$ was placed in a sterile tube where $10 \mathrm{ml}$ of erythrocyte lysis buffer (e-Bioscience) were added. After $12 \mathrm{~min}$ of incubation at room temperature (RT), tubes were centrifuged $(300 \mathrm{~g}, 5 \mathrm{~min})$ and the cell pellet was resuspended in $1 \mathrm{ml}$ of MACS buffer.

Flow cytometry immunophenotyping

We analyzed leukocyte canine antigen expression using monoclonal antibodies (mAb) from AbD (Serotec Oxford, UK): anti-canine CD3(CA17.2A12) conjugated with fluorescein isothiocyanate (FITC), anti-canine CD21(CA2.1D6) conjugated with phycoerythrin (PE). Non-conjugated antibodies CD4(YKIX302.9), and 
CD8(YCATE55.9) were supplied by the Institut für Molekulare Immunologie (München, Germany). Antibodies against tissue antigens were provided by E. Kremmer; Dog17(panT), Dog10(CD8) and anti-canine mAb 3B4 (Ostronoff et al. 2008), instead of the commercial CD34 (McSweeney et al. 1998), were incubated at $10 \mu \mathrm{g} / \mathrm{ml}$ before titration.

For the non-conjugated mAbs, $200 \mu \mathrm{l}$ of MWBCs or TWBCs samples with $1 \times 10^{6}$ cells, were placed in $5 \mathrm{ml}$ tubes and incubated for $30 \mathrm{~min}$ at $4{ }^{\circ} \mathrm{C}$ adding $10 \mu \mathrm{l}$ of primary mAbs. Cells were washed with MACS buffer (300 $\mathrm{g}, 5 \mathrm{~min})$ and then suspended in $50 \mu \mathrm{l}$ of MACS buffer. As secondary Ab PE-donkey anti-mouse-IgG was added to the sample with CD8, and FITC-rabbit anti-rat-IgG to the remaining samples. All samples were incubated for $15 \mathrm{~min}$ at $4{ }^{\circ} \mathrm{C}$ in the dark, then the cells were washed with MACS buffer $(300 \mathrm{~g}, 5 \mathrm{~min})$ and suspended in $600 \mu \mathrm{l}$ of PBS. For the conjugated mAbs, $100 \mu \mathrm{l}$ of blood or MWBCs samples (with $1 \times 10^{6}$ cells) $10 \mu \mathrm{l}$ of each antibody were added into a $5 \mathrm{ml}$ tube and incubated for $30 \mathrm{~min}$ in the dark at RT. After that, $2 \mathrm{ml}$ of erythrocyte lysis solution was added and incubated for $12 \mathrm{~min}$ at RT. Then, cells were washed with MACS buffer ( $300 \mathrm{~g}, 5 \mathrm{~min})$ and resuspended in $600 \mu \mathrm{l}$ of PBS.

Flow cytometry acquisition was performed using a FACScan (Becton Dickinson, San Jose CA, USA). Technician calibrates the FACScan everyday using the appropriate beads. A minimum of 10,000 events was acquired for each sample. Flow cytometry data were analysed using FacsDiva 6.0 software (Becton Dickinson, San Jose CA, USA). Dead cells were discarded adding $1 \mu \mathrm{g} / \mathrm{ml}$ propidium iodide (PI) (SouthernBiotech, Birmingham, USA) to the samples. Non-staining cells were used as negative controls, and secondary antibodies were used as isotype control to prevent unspecified cross staining.

Cytological evaluation

Blood smears and cytocentrifugated preparations of MWBCs and TWBCs were stained with May GrünwaldGiemsa. A minimum of 200 white blood cells was counted by two different pathologists. In flow cytometry we established the number of cells for each subpopulation; using an absolute cell counting (cell number/ $\mu 1$ of whole blood), and with the percentage gated, which is the frequency (\%) of events in the subpopulation compared with all the acquired events (Watabe et al. 2012).

Statistical analysis

Unpaired two-tailed $t$-test was performed for comparisons between two groups of non-paired data. The coefficient of variation $(\% \mathrm{CV})$ was calculated as (standard deviation $/ \mathrm{mean}) \times 100$. Statistical tests were performed on GraphPad Prism 5.0 software. $P$-values $\leq 0.05$ were considered significant.

\section{Results}

\section{Comparative analysis between differential counting and flow cytometry} in MWBCs and TWBCs

Blood samples from Beagle dogs (5 males and 5 females) were utilized during this study for comparative analysis by differential counting (DC) and flow cytometry (FC) leukocyte cell number populations utilizing two processing methods: TWBCs and MWBCs. Figure 1 (Plate VIII) shows a representative dot plot and microphotographs of both populations. Data in the columns were expressed as percentage (\%) of total cell number. The represented populations were granulocytes, monocytes and lymphocytes depending on the isolation method. We compared the number of cells selected by flow cytometry against the differential count.

Results indicated that CV between DC and FC for TWBC were 3.54, 18.92, and 18.21 for granulocytes, monocytes, and lymphocytes, respectively; 18.90 for monocytes and 6.42 for lymphocytes in MWBC. All CV values were under 25 (consensus limit for CV comparative analysis (Herzenberg et al. 2006)).

Immunophenotyping of blood leukocyte subsets in MWBCs and TWBCs

The immunophenotyping analysis was carried out in samples obtained by TWBC and MWBC. We tested the samples against an array of canine leukocyte antibodies: CD3, CD4, CD8, Dog10(CD8), CD21, Dog17(panT), and CD34. Table 1 shows the mean values of leukocyte subsets labelled with the antibodies obtained either for TWBC or MWBC. All $\mathrm{CV}$ values in the table were under 25 . Moreover, $P$-values indicated that there were no significant differences for the percentage of cells in TWBC and MWBC except for CD21. These data dealt with the main goal in this study, demonstrating that the sample preparation 
method, blood lysing (TWBC) or density gradient (MWBC), had no impact on leukocyte subpopulations for immunophenotyping.

Figure 2 (Plate VIII) shows representative dot plots from 80-90 samples of peripheral blood leukocyte subsets from samples labelled with the array of canine leukocyte antibodies for TWBC and MWBC. In the dot plots stained populations were displayed to show SSC (side scatter) vs. FITC or PE fluorescence. Our data revealed good homogeneity between samples indicating good experiment reproducibility.

Table 1. Means of cellular markers and comparative commercial vs. dog specific antibody.

\begin{tabular}{lcrrc}
\hline A & MWBC* & TWBC* & CV(n) & $P$-values \\
\hline CD3** & 58.00 & 66.10 & 6.53 & 0.1320 \\
panT $^{* *}$ & 60.60 & 71.80 & 8.46 & 0.0904 \\
CD4** & 37.00 & 38.75 & 2.31 & 0.0592 \\
CD8** & 23.00 & 16.70 & 15.87 & 0.0871 \\
Dog10** & 16.80 & 19.50 & 7.44 & 0.4295 \\
CD21** & 13.65 & 20.45 & 19.94 & $0.0211^{*}$ \\
3B4*** & 0.55 & 0.91 & 24.63 & 0.9191 \\
B & & & & \\
CD3/panT MWBC & & & & 0.9472 \\
CD3/panT TWBC & & & & 0.4747 \\
CD8/Dog10 MWBC & & & & 0.0766 \\
CD8/Dog10 TWBC & & & & 0.6891 \\
\hline
\end{tabular}

A: Mean values of immunophenotype from peripheral blood leukocyte subsets obtained using the two different methodologies, density gradient separation of mononuclear cells and whole blood lysis. CV was obtained after comparative analysis of mean values from $\mathrm{MWBC}$ and TWBC for each antibody; all CV values are below 25 . Included are the results of the statistical analysis unpaired two-tailed $t$-test for each antibody; there are no significant differences $(P>0.05)$ between leukocyte subsets and ratios, with the exception of CD21.

* Mean values are expressed in \%

** Data are referred to lymphocyte population

*** Data are referred to total live cells

B: Comparative results between CD3 versus the tissue antigen Dog 17 (panT) and CD8 versus the tissue antigen Dog10 (CD8).

\section{Storage of samples of canine blood cells}

The effects of storage of blood leukocyte samples, depicted in percentages of leukocyte subpopulations and immunophenotyping pattern, were investigated on peripheral TWBC. We carried out a comparative long-term stability analysis at two time points: TWBC immediately processed $(0 \mathrm{~h})$ or stored $48 \mathrm{~h}$ at $4{ }^{\circ} \mathrm{C}$. Data analysed at $0 \mathrm{~h}$ were previously presented in (Plate VIII, Fig 1).

After $48 \mathrm{~h}$ of storage at $4{ }^{\circ} \mathrm{C}$, TWMC samples were analysed to determine leukocyte subpopulations using the two methods; FC and DC. Figure 3A shows the leukocyte subpopulation at $48 \mathrm{~h}$ of storage. The $\mathrm{CV}$ values were $2.60,21.75$, and 0.74 for granulocytes, monocytes and lymphocytes, respectively. Data of cell population were represented as means with an error bar. In (Plate IX, Fig. 3) we analysed subpopulations at two different time points, $0 \mathrm{~h}$ vs. $48 \mathrm{~h}$, for FC Fig. 3B and for DC Fig. 3.C as intra-method comparison. In both cases, $P$-values indicated no significant differences between the samples due to storage.

We then carried out an immunophenotype analysis at two time points (Plate IX, Fig. 4). Our data showed no significant differences to any of the Ab used for this study.

Our study was performed with healthy dogs of both sexes. We carried out a comparative analysis between males and females using the cell number in TWBC samples. Our study 
showed a CV of 5.86, 5.80, and 0.99 for granulocytes, monocytes and lymphocytes, respectively. Also we evaluated the immunophenotyping of $\mathrm{T}$ (pan $\mathrm{T}$ ) and $\mathrm{B}$ (CD21) lymphocytes in both sexes finding a CV of 4.39 for pan $\mathrm{T}$ and 19.88 for CD21. The B-lymphocytes showed a $20 \%$ of variance. Also, no significant differences were observed between male and female donors, $P$-values were $P=0.19, P=0.19$, and $P=0.15$ for granulocytes, monocytes, and lymphocytes, respectively, and $P=0.11$ for T-lymphocytes and $P=0.18$ for B-lymphocytes.

\section{Discussion}

Clinical applications of flow cytometry have become common practice to establish ranges for phenotype percentages in healthy animals; this is essential as reference data to monitor alterations associated with disease. In this study, leukocyte subsets were examined in 7.5-year-old Beagle dogs, using whole blood lysis or density gradient centrifugation to obtain cell populations. We determined that those two methods could be equally used for animal diagnosis. To the best of our knowledge and despite the broad usage of the two methods in immunophenotyping, only little comparative information is available to date. Moreover, this is a long-term stability study in terms of information on leukocyte subpopulations and analysis of antibodies. Often, blood samples are collected at one site and then shipped to another site for analysis.

Here we demonstrated that no significant differences were found between samples processed following a whole-blood lysis method and samples processed after density gradient separation (Plate VIII, Fig. 1 and Table 1). It is important also to indicate that our immunophenotyping data of lymphocytes were within the same range as those obtained for 8-year-old healthy dogs described previously (Williams et al. 2008), and for dogs over 5 years of age (Faldyna et al. 2001).

No differences in canine leukocyte subpopulations have been described in the literature between males and females although Faldyna et al. (2001) concluded that perhaps there were sex-related differences in the lymphocyte subsets they studied, even if these differences were examined in Beagles and Dachshunds 1-5 years old. However, our results should be interpreted carefully taking into account the limited number of animals included in each group. The results of this part of the study confirmed our preliminary hypothesis based on literature, relating the lack of differences between cell populations and leukocyte subsets when using donors of different sex.

In our study, we compared results obtained with the commercial antibody CD3 versus the specific Dog17(panT) and data obtained with the commercial CD8 versus Dog10 (CD8), (Table 1B) with no significant differences between values either for MWBC or TWBC. These findings allow us to validate the use of Dog17 as a panT marker, and the Dog10 as CD8 antibody specifics for dogs.

As we indicated previously this paper offers a panel of canine antibodies used in healthy dogs, which will be helpful to estimate levels of antibodies in future studies of canine leukaemia or malignant lymphoma. In that respect, and taking into consideration the amount of B-associated neoplasias, we will plan to amplify the B lymphocyte panel of antibodies, which are critical to identify and classify those diseases.

As far as we know, no methodology has been published focusing on the long-term ( $48 \mathrm{~h}$ ) sample storage on canine leukocytes. Our results demonstrated that there are no significant differences at different time points $(0 \mathrm{~h}$ and $48 \mathrm{~h})$. That indicates a great advantage for laboratory workers or for the processing and also the possibility to store samples without any disturbance in the cell populations, independently of the method used for cell analyses, FC or DC. Furthermore, the same results were observed when we studied antigen expression of samples immediately processed and analysed after blood collection $(0 \mathrm{~h})$ and sample 
storage for $48 \mathrm{~h}$ at $4{ }^{\circ} \mathrm{C}$. Figure 4 shows the $P$-values. Our results indicate that storage at $4{ }^{\circ} \mathrm{C}$ for $48 \mathrm{~h}$ had no effect on leukocyte subset distribution. In concordance with our data, other authors (Shield et al. 1983; Nicholson et al. 1984; Ponzio et al. 1984; Prince et al. 1986) describe similar conclusions for storage for a shorter period of time, between $2-16 \mathrm{~h}$.

Since Faldyna et al. (2001) described the difficulties in monocyte determination only by their light scatter characteristics in flow cytometry, several new flow cytometry analysis softwares have been released. Here, we gated a monocyte population using flow cytometry with accurate precision when comparing the monocyte flow cytometry data with differential counting data, with CV less than 20.

The outcome of the present study showed that whole blood lysis represents an efficient and quick alternative for canine leukocyte preparation. In addition, samples can be stored $48 \mathrm{~h}$ at $4{ }^{\circ} \mathrm{C}$ without a significant difference with respect to samples processed immediately after blood collection. This finding is relevant for veterinary medicine considering the lack of facilities in many laboratories to do immunophenotyping studies. These results indicate that whole blood lysis represents an effective, fast and cheap alternative for canine leukocyte preparation.

\section{Conflict of interest}

The authors declare no conflict of interest.

\section{Acknowledgements}

Leticia G. León thanks her contract to the European grant FP7-REGPOT-2012-CT2012-31637-IMBRAIN. The authors thank Santiago Viñambres for his excellent technical assistance.

\section{References}

Cobbold S, Metcalfe S 1994: Monoclonal antibodies that define canine homologues of human CD antigens: summary of the First International Canine Leukocyte Antigen Workshop (CLAW). Tissue Antigens 43: 137-154

De Paoli P, Reitano M, Battistin S, Castiglia C, Santini G 1984: Enumeration of human lymphocyte subsets by monoclonal antibodies and flow cytometry: a comparative study using whole blood or mononuclear cells separated by density gradient centrifugation. J Immunol Methods 72: 349-353

Faldyna M, Levá L, Knötigová P, Toman M 2001: Lymphocyte subsets in peripheral blood of dogs-a flow cytometric study. Vet Immunol Immunopathol 82: 23-37

Hardy MY, Vari F, Rossetti T, Hart DN, Prue RL 2013: A flow cytometry based assay for the enumeration of regulatory $\mathrm{T}$ cells in whole blood. J Immunol Methods 390: 121-126

Herzenberg LA, Tung J, Moore WA, Herzenberg LA, Parks DR 2006: Interpreting flow cytometry data: a guide for the perplexed. Nat Immunol 7: 681-685

Kolb HJ, Günther W, Schumm M, Holler E, Wilmanns W, Thierfelder S 1997: Adoptive immunotherapy in canine chimeras. Transplantation 63: 430-436

Krause DS, Fackler MJ, Civin CI, May WS 1996: CD34: structure, biology, and clinical utility. Blood 87: 1-13

Mansour I, Bourin P, Rouger P, Doinel C 1990: A rapid technique for lymphocyte preparation prior to two-color immunofluorescence analysis of lymphocyte subsets using flow cytometry. Comparison with density gradient separation. J Immunol Methods 127: 61-70

McSweeney PA, Rouleau KA, Wallace PM, Bruno B, Andrews RG, Krizanac-Bengez L, Sandmaier BM, Storb R, Wayner E, Nash RA 1998: Characterization of monoclonal antibodies that recognize canine CD34. Blood 91: 1977-1986

Nicholson JK, Jones BM, Cross GD, McDougal JS 1984: Comparison of T and B cell analyses on fresh and aged blood. J Immunol Methods 73: 29-40

Ostronoff LK, Kremmer E, Fermín ML, Fragío C, Mysliwietz J, Kolb HJ, Tejero C 2008: Canine stem cell factor augments expression of matrix metalloproteinase-9 by CD34 cells. Cytotherapy 10: 193-202

Owens MA, Vall HG, Hurley AA, Wormsley SB 2000: Validation and quality control of immunophenotyping in clinical flow cytometry. J Immunol Methods 243: 33-50

Ponzio AD, Self SE, Runey DH, Burdash NM, La Via MF 1984: Are lymphocyte subset determinations affected by storage conditions? Diagn Immunol 2: 188-190

Prince HE, Arens L 1986: Effect of storage on lymphocyte surface markers in whole blood units. Transplantation 41: $235-238$ 
Schwonzen M, Diehl V, Dellanna M, Staib P 2007: Immunophenotyping of surface antigens in acute myeloid leukemia by flow cytometry after red blood cell lysis. Leuk Res 31: 113-116

Shield CF 3rd, Marlett P, Smith A, Gunter L, Goldstein G 1983: Stability of human lymphocyte differentiation antigens when stored at room temperature. J Immunol Methods 62: 347-352

Van Dongen JJ, Langerak AW, Brüggemann M, Evans PA, Hummel M, Lavender FL, et al 2003: Design and standardization of PCR primers and protocols for detection of clonal immunoglobulin and T-cell receptor gene recombinations in suspect lymphoproliferations: report of the BIOMED-2 Concerted Action BMH4-CT98-3936. Leukemia 17: 2257-2317

Watabe A, Hanazono K, Komatsu T, Fu DR, Endo Y, Kadosawa T 2012: Peripheral lymphocyte subsets as a prognostic indicator of mortality and morbidity in healthy dogs. J Vet Med Sci 74: 937-943

Weiss DJ 2002: Application of flow cytometric techniques to veterinary clinical hematology. Vet Clin Pathol 31: $72-82$

Williams DL 1997: Studies of canine leucocyte antigens: a significant advance in canine immunology. Vet J 153: 31-39

Williams MJ, Avery AC, Lana SE, Hillers KR, Bachand AM, Avery PR 2008: Canine lymphoproliferative disease characterized by lymphocytosis: immunophenotypic markers of prognosis. J Vet Intern Med 22: 596-601

Zeigler BM, Boyle-Holmes Y, Falzone D, Farmer J 2013: Validation of an eight parameter immunophenotyping panel in adult canines for assessment of immunotoxicity. Vet Immunol Immunopathol 154: 75-81 

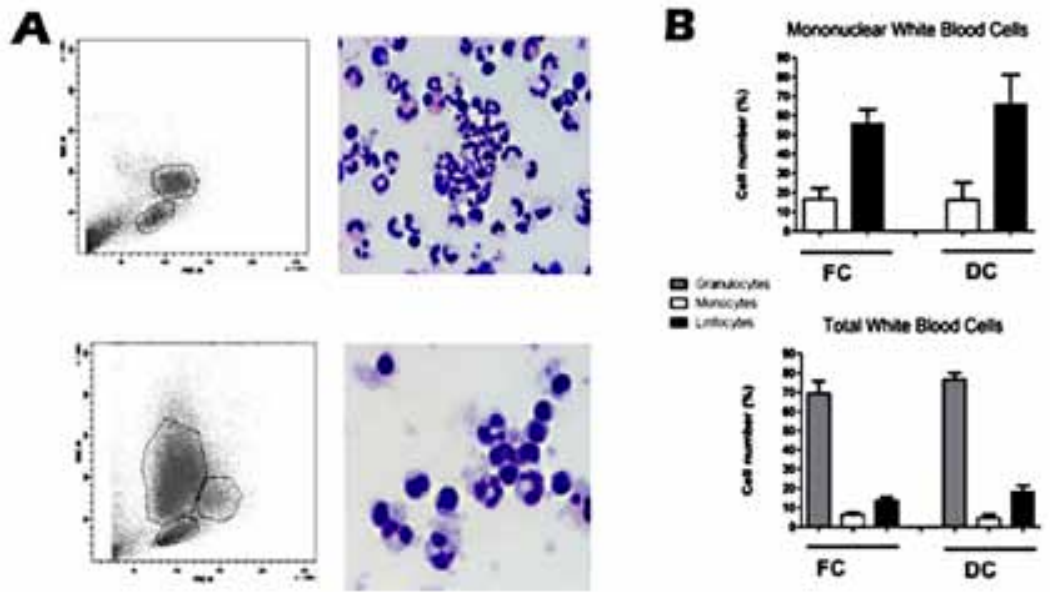

Fig. 1. MWBC and TWBC populations

A: Representative dot plots (85-90 different samples) of cell populations obtained using whole blood lysis or density gradient centrifugation. In parallel with these dot-plots are included microphotographs $(\times 400)$ B: Data from comparative analysis between flow cytometry (FC) and differential count (DC) are represented in the bar graphs. The bars represent the $\%$ of the mean and error bar for the different populations.

MWBC: mononuclear white blood cells, (monocytes and lymphocytes); TWBC: total white blood cells, (granulocytes, monocytes, and lymphocytes).

Comparative analysis indicated a coefficient of variation (CV) of 18.90 and 6.42 for monocytes and lymphocytes, respectively, in MWBC, and a CV of 3.54, 18.92, and 18.21 for granulocytes, monocytes, and lymphocytes, respectively, in TWBC.

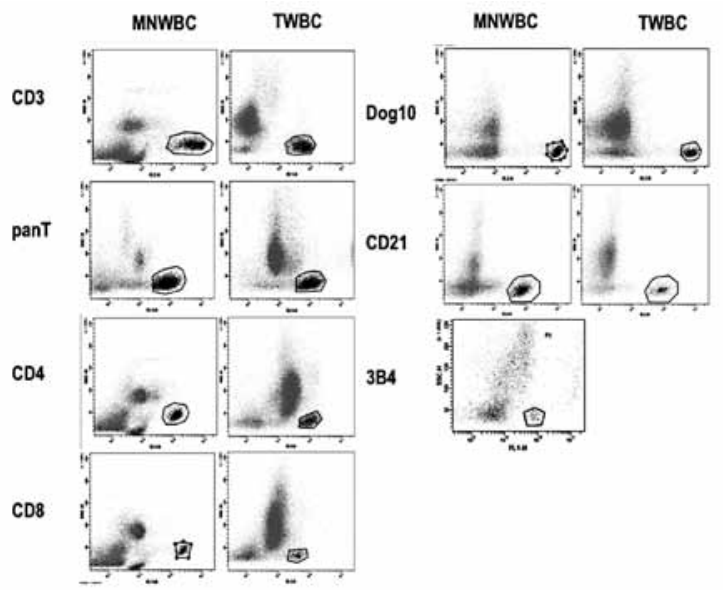

Fig. 2. Representative dot plots of peripheral blood leukocyte subsets.

Representative dot plots (80-90 different samples) of peripheral blood leukocyte subsets. The figure shows flow cytometry analysis for the cell population stained by different antibodies. The gating was made taking in consideration previous data used for this study. 10,000 cells were acquired for each sample. Cell dead discrimination was make by forward and side scatter (FSC/SSC) and PI. 

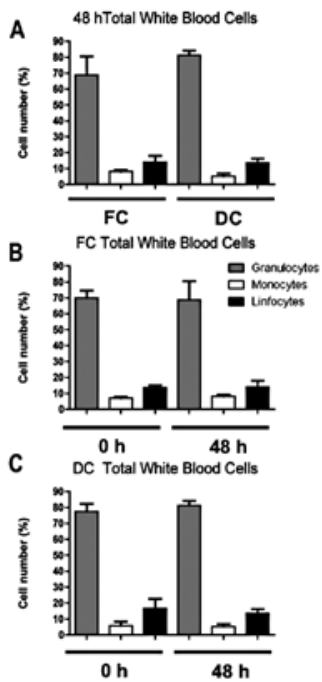

Fig. 3. TWBC time stability

This figure shows long-term stability of total white blood cells (TWBC) at two time points. Data in the column are represented by mean and error bar. A: Comparative analysis was made between flow cytometry (FC) and differential count (DC) at $48 \mathrm{~h}$. B: Analysed subpopulations at two different time points $(0 \mathrm{~h}$ vs $48 \mathrm{~h})$ using $\mathrm{FC}$ as control. C: Analysed subpopulations at two different time points $(0 \mathrm{~h}$ vs 48 h) using DC. For both methods, FC (Fig. 3B) and DC (Fig. 3C), the $P$-values obtained from comparison of the two time points give no significant differences $(P>0.05)$ in any cell population.
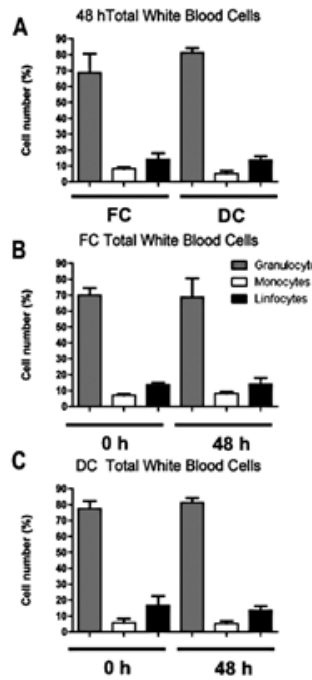

Fig. 4. Population stability: leukocyte subsets data at different time points

The mean and error bar represent data in the column graphs. Mean values of the immunophenotype from total white blood cell leukocyte subsets at two time points $0 \mathrm{~h}$ and $48 \mathrm{~h}$. The statistical analysis (unpaired two-tailed $t$-test) for the $\mathrm{FC}$ data at the two time points, as well as the DC, data reveal no significant differences $(P>0.05)$ in the percentage of leukocyte subsets (data not shown). 